\title{
3D Spatial Mapping of the Nanomorphology of Polymer:Fullerene Blends by Highly Selective, Homogeneous Copper Staining
}

Yonghe $\mathrm{Li}^{1}$, Martin Čalkovský ${ }^{1}$, Erich Müller ${ }^{2}$, Christian $\mathrm{Sprau}^{3}$, Alexander Colsmann ${ }^{4}$ and Dagmar Gerthsen $^{5}$

${ }^{1}$ Laboratory for Electron Microscopy, Karlruhe Institute of Technology, United States, ${ }^{2}$ Karlsruhe Institute of Technology (KIT), Laboratory for Electron Microscopy (LEM), Engesserstraße 7, 76131 Karlsruhe, Germany, United States, ${ }^{3}$ Light Technology Institute,Karlsruhe Institute of Technology, United States, ${ }^{4}$ Light Technology Institute, Karlsruhe Institute of Technology, United States, ${ }^{5}$ Laboratorium für Elektronenmikroskopie, Karlsruher Institut für Technologie (KIT), Engesserstr. 7, 76131 Karlsruhe, Germany, United States

Bulk-heterojunction absorber layers of organic solar cells (OSCs) consist of three-dimensional (3D) interpenetrating networks of conjugated polymer and fullerene (donor/acceptor) domains [1]. The power conversion efficiency of OSCs depends strongly the intrinsic physical properties such as the exciton diffusion length and the charge carrier dissociation at interfaces of donors and acceptors. It requires a nanomorphology with domains sizes that are on the order of the exciton diffusion length. Thus, the nanomorphology has to be carefully characterized for correlation with solar cell performance. However, owing to their similar material properties, distinction of conjugated polymers and fullerenes in bulkheterojunctions is always hampered by low contrast in electron microscopy. A common method for contrast enhancement is selective staining where one phase of a material mixture is labeled by heavier elements. This increases the difference of the average atomic number $\mathrm{Z}$ and yields $\mathrm{Z}$-contrast imaging. Staining agents like $\mathrm{OsO}_{4} / \mathrm{RuO}_{4}$,[2] and uranylacetate [3] are commonly used. However, the staining process can lead to swelling of nanoscaled phases, and, more seriously, these staining agents are highly toxic.

To overcome these issues, we describe in this work a facile procedure for highly selective and homogeneous $\mathrm{Cu}$ infiltration that can be used as staining procedure to recognize the phase distribution in sulfur-containing conjugated polymer:fullerene absorber layers in OSCs. Figure 1a shows a high-angle annular dark-field scanning transmission electron microscopy (HAADF-STEM) image of a focused-ionbeam (FIB-) milled TEM lamella of a model sample consisting of pure $\mathrm{PC}_{61} \mathrm{BM}\left(\mathrm{C}_{72} \mathrm{H}_{14} \mathrm{O}_{2}\right), \mathrm{P} 3 \mathrm{HT}$ $\left(\mathrm{C}_{10} \mathrm{H}_{14} \mathrm{~S}\right), \mathrm{PC}_{71} \mathrm{BM}\left(\mathrm{C}_{82} \mathrm{H}_{14} \mathrm{O}_{2}\right)$ and $\mathrm{PTB} 7\left(\mathrm{C}_{41} \mathrm{H}_{53} \mathrm{FO}_{4} \mathrm{~S}_{4}\right)$ layers. The lamella was attached to a $\mathrm{Cu}$ liftout grid. The image hardly shows any contrast between the layers apart from thin PEDOT:PSS layers which are located between different layers of the stack (blue arrows) as remnants of the sample-fabrication process. The contrast between layers is drastically enhanced after thermal treatment at $200{ }^{\circ} \mathrm{C}$ for $3 \mathrm{~h}$ in air where conjugated polymer appear with high intensity (Figure 1b). In addition, Figure 1a,b show the $\mathrm{S}$ and $\mathrm{Cu}$ distributions obtained by energy dispersive X-ray spectroscopy (EDXS) mapping. We find a homogenous $\mathrm{Cu}$ distribution after thermal treatment that correlates with the S distribution in PTB7, P3HT and PEDOT:PSS. The source of the $\mathrm{Cu}$ infiltration is the $\mathrm{Cu}$ lift-out grid. High-resolution TEM images (not shown here) demonstrate that $\mathrm{Cu}$ is homogeneously dissolved in the polymers without the formation of clusters or crystalline nanoparticles. Fullerene derivatives ( $\mathrm{PC} 61 \mathrm{BM}$ and $\mathrm{PC} 71 \mathrm{BM})$ do not contain any sulfur and are not infiltrated by $\mathrm{Cu}$. The new selective staining technique is not only suitable for HAADFSTEM imaging but can be also used for secondary-electron scanning electron microscopy (SE-SEM) imaging. This facilitates mapping of the domain structure of polymer:fullerene absorber layers in OSCs 
in a cross-section perspective and subsequent reconstruction of the 3D nanomorphology by FIB-SEM tomography.

For this purpose, prototype $\mathrm{PTB} 7: \mathrm{PC} 71 \mathrm{BM}$ absorber layers processed with different additives, which are commonly employed to tailor the nanomorphology of bulk-heterojunctions. As an example we present the nanomorphological investigation of the absorber layer processed with 3\% p-anisaldehyde (AA). A thick TEM cross-section lamella was prepared by FIB-milling, which was attached to a Cu lift-out grid. Figure 2a shows SE-SEM images of two stacked absorber layers separated by a bright PEDOT:PSS layer in cross-section perspective before and after thermal treatment. PTB7 and PC 71BM cannot be distinguished in the pristine state. After thermal treatment, $\mathrm{Cu}$ staining strongly enhances the SE-SEM intensity of Scontaining polymer domains, which allows to clearly differentiate materials and measure domain sizes. Figure 2b shows the two stained absorber layers embedded in a layer stack with pure PTB7 and PC 71 BM layers. The latter yield reference intensities for the pure compounds and even allow to distinguish local PTB7-rich and PC71BM-rich phases at intermediate SE-SEM intensities. After segmentation, the distribution of different domains is indicated by the color coding on the absorber layers in Figure $\mathbf{2 b}$. Irregular-shaped pure $\mathrm{PC}_{71} \mathrm{BM}$ domains (dark red) with comparably large sizes of 100-200 nm are formed, which are embedded in PC71BM-rich (green) and PTB7-rich (purple) regions. Only narrow pure PTB7 domains (yellow) at the bottom of the absorber layers are observed.

The 3D reconstruction of the domain structure provides more information than 2D images on the interconnectivity and morphology of the absorber layer. Thus, FIB-SEM tomography was performed by alternating FIB milling and SE-SEM imaging (see scheme in Figure 2c) leading to a stack of images for $3 \mathrm{D}$ reconstruction. The blue color in the reconstructions in Figure 2d represent pure $\mathrm{PC} 71 \mathrm{BM}$ phase while mixture and possible pure PTB7 phase are transparent. Different perspectives of the total reconstructed volume (thickness $\sim 0.47 \mathrm{~mm}$ ) show irregular-shaped and well-connected PC71BM domains. The 3\% AAprocessed blend exhibits a rather coarse $\mathrm{PC} 71 \mathrm{BM}$ domain structure with sizes in the 100 to $500 \mathrm{~nm}$ range.

To summarize, a new selective staining method has been developed leading to selective $\mathrm{Cu}$ staining of Scontaining polymers with a $\mathrm{Cu}$ lift-out grid as $\mathrm{Cu}$ source. The resulting strong contrast between $\mathrm{S}$ containing polymers and fullerene derivatives is the prerequisite for the visualization of the domain structure with high contrast and for 3D reconstruction of the nanomorphology of polymer:fullerene absorber layers. We expect the stimulation of more studies of metal staining for phase identification in block copolymers and OSCs candidates and even studies how metal infiltration can be used to tune the electronic and optical properties in organic semiconductors [4]. 

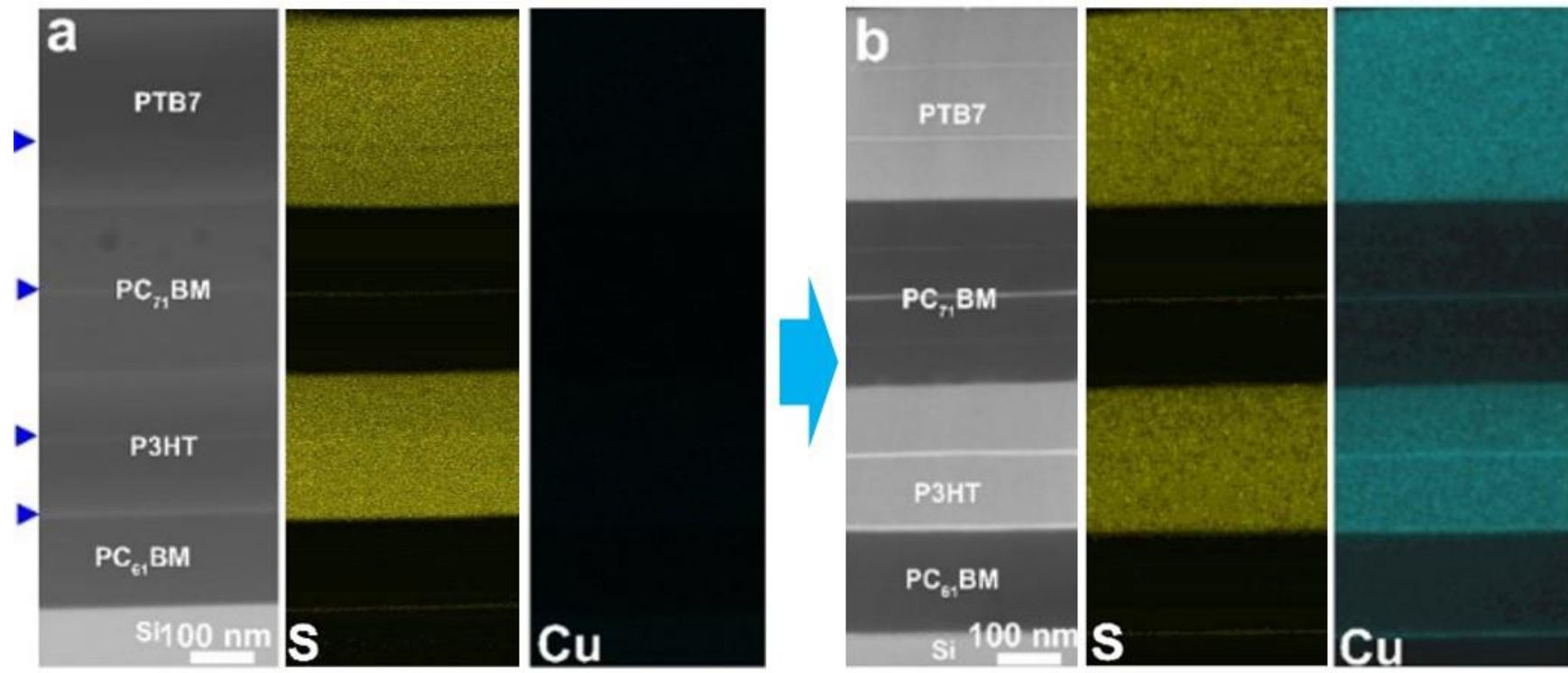

Figure 1. Observation of a selective $\mathrm{Cu}$ distribution in $\mathrm{S}$-containing polymer layers. Comparison of $200 \mathrm{keV}$ HAADF-STEM image and S/Cu distributions of the PTB7/PC71BM/P3HT/PC61BM multilayer stack on a Si substrate in the (a) as-prepared TEM lamella and (b) after thermal treatment at $200 \mathrm{oC}$ for $3 \mathrm{~h}$ in air.
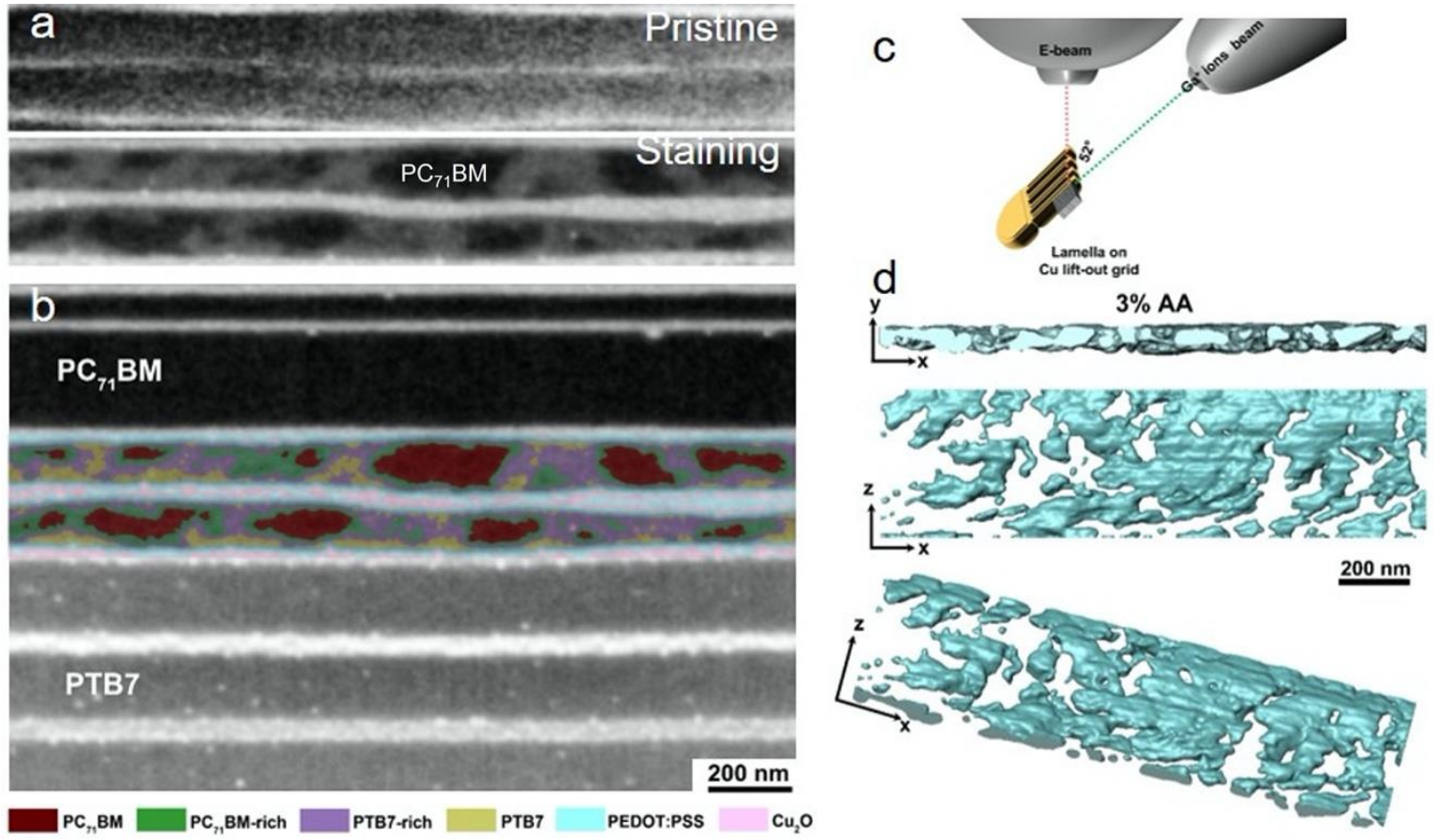

Figure 2. Application of selective $\mathrm{Cu}$ staining in PTB7:PC71BM absorber layers that were fabricated with 3\% AA as processing additive. (a) $2 \mathrm{keV}$ SE-SEM cross-section images of PTB7:PC71BM absorber layers in the pristine state and after thermal annealing. (b) $2 \mathrm{keV}$ SE-SEM cross-section images of PTB7:PC71BM absorber layers in a multi-layer stack with pure PC71BM and PTB7 layers as reference for phase segmentation. Pure PTB7 and PC71BM, as well as PTB7-rich and PC71BM-rich mixtures are color coded (see legend). (c) Scheme of the experimental setup for FIB-SEM tomography and (d) 3Drendered reconstruction of the domain structure with PC71BM domains in light blue. Mixture and pure PTB7 are transparent. 


\section{References}

[1] Heeger, A. J., Adv. Mater. 26 (2014), p. 10-28.

[2] Zheng, H., Kim, K., Kravchenko, A., et al., Environ. Sci. Tech. 54, 2020, p. 8980-8989.

[3] Harris, J. R., Horne, R. W. Micron 25,1994, p. 5-13.

[4] We gratefully acknowledge the support of Alexander von Humboldt Fellowship. 